\title{
Experimental Investigation and Numerical Analysis on Horizontal Axis Wind Turbine with Winglet and Pitch Variations
}

\author{
Nyoman Ade Satwika*, Ridho Hantoro, Sarwono Sarwono, Gunawan Nugroho, \\ Department of Engineering Physics, Faculty of Industrial Engineering, Institut Teknologi Sepuluh \\ Nopember, Surabaya 60111, Indonesia \\ *E-mail: anyomanadee@gmail.com (Corresponding author),
}

\begin{abstract}
HAWT with three blades often used because it has the highest coefficient of performance among other turbines. The airfoil used is Clark-Y type because it has a high glide ratio (coefficient lift/coefficient drag) in the application of subsonic flow. The main purpose of this study is to increase the power coefficient value obtained by the increase of lift force on each airfoil of blade compiler and to fix the wind turbine performance. One of the variations added is the addition of winglets on the tip of the blade. The method employed is a laboratory scaled experiment by using wind tunnel. Theoretically, this study also applied blade element momentum (BEM) as the calculation of each segment on the airfoil, and the simulation was carried out with computational fluid dynamics (CFD), in order to find out the characteristics of flow passing by the rotor. The advantage of using winglets is, fostering the condition of starting wind turbine rotor on low tip speed ratio (TSR) condition by varying the pitch angle on the blade. The addition of pitch variation gives an advantage that it can maximize the wind speed towards the angle of attack to the airfoil; hence, it increases the aerodynamic effect on the rotor.
\end{abstract}

Keywords: Horizontal axis wind turbine, Winglet, experiment, BEM, CFD.

ENGINEERING JOURNAL Volume 23 Issue 6

Received 21 October 2018

Accepted 18 October 2019

Published 30 November 2019

Online at http://www.engj.org/

DOI:10.4186/ej.2019.23.6.345 


\section{Introduction}

The wind turbine type used until now classified into two categories, namely horizontal axis wind turbine (HAWT) and vertical axis wind turbine (VAWT). The horizontal axis wind turbine (HAWT) has a number of advantages compared to the vertical axis wind turbine. HAWT has rotor angle which functions to rotate the turbine, in order to produce the aerodynamic effect to rotate the rotor, which synchronized with the generator, to produce electrical power. The rotor angle is rotating because of the forces working around it, namely the lift and drag forces [1]. The lift force makes the turbine rotating, while the drag force is the inhibitor force makes the rotating speed of the turbine decreased. It caused by the reverse flow to the rotor. Reducing the drag force on wind turbines can be done by using winglets. The winglets reduce vortex that occurs on the tip of the blade [2]-[5]. The addition of the device on HAWT, that is adding winglets on the tip of the blade is able to increase the performance by splitting the flow on the tip as well as to give positive effect towards the vortex reduction [6]-[9].

The winglet type selection on the blade turbine affect the produced performance, thus winglets type adaptation towards the geometry of the blades are needed, hence there is no significant change in the ratio aspect on the blade geometry towards the addition of winglets, which affects the increase of drag profile and losing the energy in the system [7]. The paper referred in [5] giving the result in the form of winglets addition on the part of the blade gives a better performance on the rotor than the blade without winglets, because the addition of winglets on the blade in this study is able to stimulate the lift force resulted from the vortex which is able to be generated. According to these studies from [10]-[12], they explain that the addition of pitch angle variations on HAWT aims at serving as torque starting, that is the angle used for rotating the turbine initially because of the on the low wind speed, it results from high torque.

In addition, it applied to low TSR. The pitch angle is able to be used as a brake or rotating adjustment as well in order to be constant because by adjusting the pitch angle on the bigger angle, whereas tip speed ratio (TSR) is comparison between the tangential velocity of the tip blade and the actual velocity from the wind, the TSR will become low. The previous study from [13] about the comparison of the result of Q-blade simulation and CFD in terms of design optimization on the wind turbine, mentioned that the use of Q-blade has the higher value of aerodynamic effect performance than the use of CFD. Q-blade. Nevertheless, it cannot discuss the visualization on Q-blade about the characteristics of the working flow resulted on the blade turbine rotor. There are three types of analysis based on the simulation and theoretical calculation. The simulation used was CFD, aiming at figuring out the pressure distribution occurred on the blade when blended winglets added and the analysis of flow characteristics behind the rotor. The method of theory simulation was done by using blade element momentum (BEM), which served as the experiment result in comparison from the method of blade enumeration into a number of segments hence the optimum pitch angle value can be determined based on the lift and drag coefficient values gained from a number of segments the blade. Therefore, it figured out that the data used for finding out the characteristics of the performance of variations were determined on the system [15].

The result shows that the configuration of pitch angle adjustment results from simplicity in terms of the adjustment of the effective angle of attack on the highest value of glide ratio $(\mathrm{Cl} / \mathrm{Cd})$, which glide ratio is the infenctiont of the continue velocity devided by sink velocity on the airfoil. The effectiveness of lift force is higher than the increase of produced drag force, and also the addition of winglet on the tip of the turbine blade gives good performance when it is working in low TSR

\section{Designing and Constructing the Winglets of The Horizontal Axis Wind Turbine}

The design of the blade refers to the previous study [16], [17]. The selection of three blades on the HAWT design based on the graphics of turbine characteristics with a maximum coefficient of performance $(C p)$. Coefficient of performance $(C p)$ is comparison between electricity produced by wind turbine devided by total energy availaible in the wind at the velocity. The selection of airfoil type is done by using Clark-Y because it has a high glide ratio or $\mathrm{Cl} / \mathrm{Cd}$ compared to other airfoils. It indicates that the airfoil will be easier to lifting. In designing the shape of the blade, chord $(c)$ value and twist angle $(\beta)$ is needed. There are two distributions to calculate optimization of the chord length and twist angle, namely Betz and Schmitz distributions [18]. The distribution used in this study is Schmitz distribution because it has the shape of chord distribution and twists angle, it because is easy to be made and result from high solidity value. The formula equation of Schmitz distribution is described as follows: 


$$
\begin{aligned}
& c(r)=\frac{16 \pi r}{B C_{l}} \sin ^{2}\left(\frac{1}{3} \tan ^{-1}\left(\frac{R}{\lambda r}\right)\right) \\
& \beta(r)=\frac{2}{3} \arctan \frac{R}{r \lambda}-\alpha_{D}
\end{aligned}
$$

Remark: $r \quad$ Radius of the blade $(\mathrm{cm})$

$B \quad$ Number of the blade

$C_{L} \quad$ Coefficient lift on airfoil

$\mathrm{R} \quad$ Diameter of the rotor $(\mathrm{cm})$

$\lambda \quad$ Maximum tip speed ratio on airfoil

$a_{D} \quad$ Angle of attack at optimum glide ratio $\left({ }^{\circ}\right)$

16 segments are presented on the blade design by using Schmitz distribution. There are three main variables in main blade design in order to encompass radius, chord, and twist. The blade construction process begins from blade and hub designing using solidworks.

The geometry converted in the form of stereolithography (STL). This storage in the form of STL eventually printed by using 3D printing machine in full high definition (HD) quality.

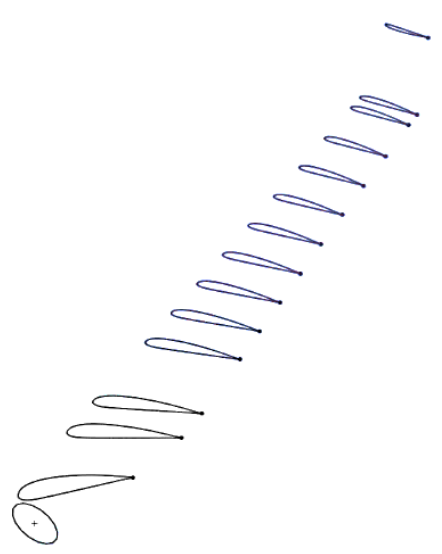

(a)

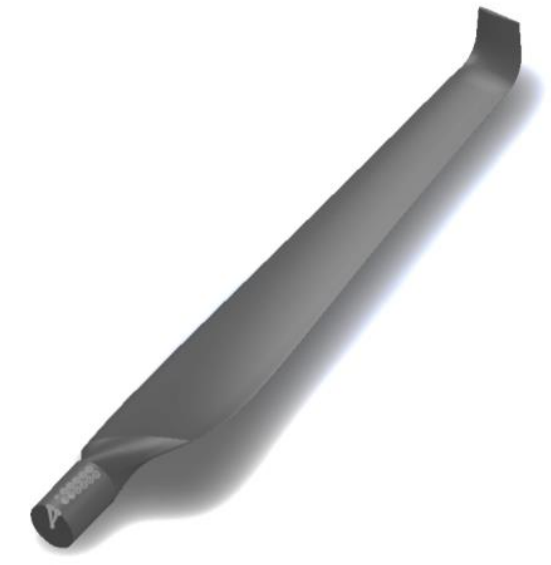

(b)

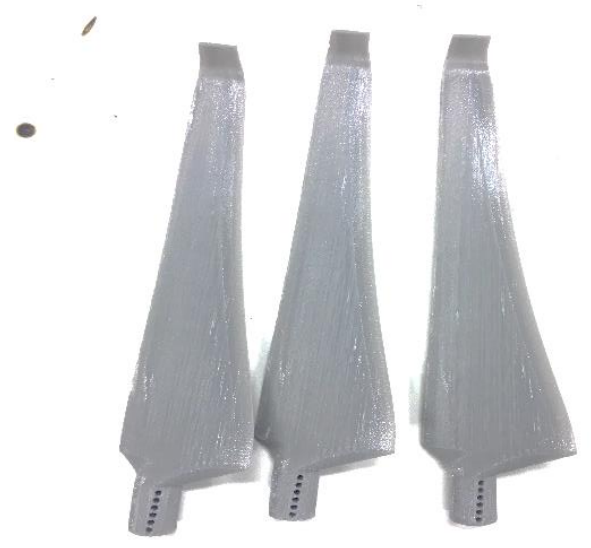

(c)

Fig. 1. Blade design with 16 Segmentsusing Schmitz method (a), loft process on a blade (b), and the fabrication 3D printing on the blade (c). 
Figure 1 is the arrangement of the total segment that used on the blade with winglet designing. The total segment used was 16, in order to the arrangement on the blade used Clark-Y airfoil and for the winglet, the airfoil NACA 0012 [16] was used. The determined airfoil position on the blade arrangement correlated on the used blade dimension, the radius of each blade, twist, the length of the chord, and the center of gravity.

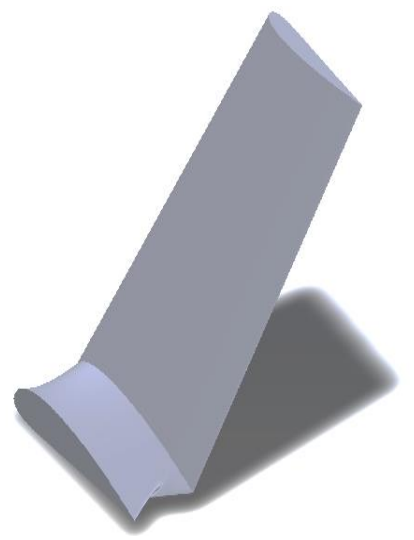

(a)

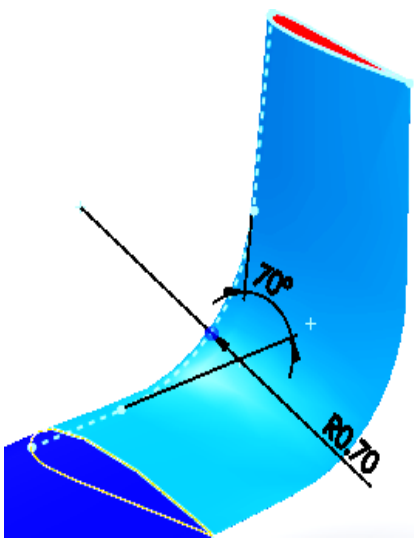

(b)

Fig. 2. Winglet design.

The blended winglet is used as the type of winglet which will be designed as high lift device on the blades. The steps of designing were treated almost the same with the blades without winglets, thus the difference merely lies on the winglet angle adjustment and the addition of segment as winglet segment.

It is similar with blade designing; solid works was used on winglet designing, with a few parameters, which include cant angle, with the angle of $70^{\circ}$ and winglet length of $1.5 \mathrm{~cm}$.

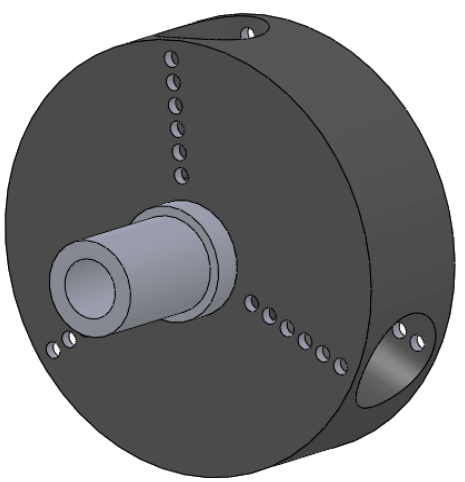

(a)

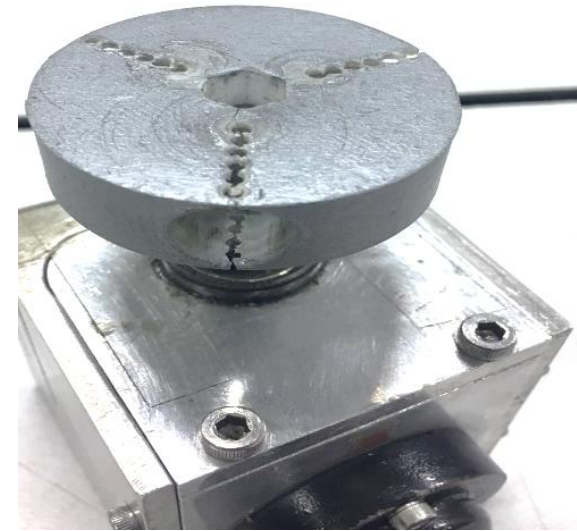

(b)

Fig. 3. The variation pitch angle on hub turbine.

The hub turbine design adapts the pitch angle variation. There are six holes on each turbine shaft. It represents used pitch, with the variation of $0^{0}, 2^{0}, 4^{0}, 6^{0}, 8^{0}$, and $10^{\circ}$. The diameter of the pitch hole is $2 \mathrm{~mm}$, which is functioned as the blade lock during the test.

\subsection{The Analysis of Two Dimensions using Blade Element Momentum (BEM)}

The calculation of blade element momentum (BEM) aims at determining the aerodynamic characteristics. It obtained during the two dimension designing, through the categorization of a number of segments on turbine blade with the iteration process. The aim of this iteration is to obtain the value of the axial induction factor (a) and tangential ( $a$ ). After obtaining $a$ and $a$ 'values which are convergent during the process of iteration, then it can be continued to the torque calculation on each segment and blade torque [21], [27], [28]. The blade 
torque will multiple with the total blade, hence the power value on the rotor can figure out as well as the coefficient of performance $(C p)$ value.

BEM simulation carried out based on the numerical calculation of each segment of the blade arrangement. The calculation and data processing is done in Excel, with few inputs which encompass geometry design, test variations, as well as the properties of the air in the calculation process.

\subsection{Experimental Setup on Wind Tunnel}

The Experimental process on HAW'T carried out on the wind tunnel, where the rotor of the wind turbine is set on the part of the test section. Before entering the fan, there is a nozzle section as the connector of the test section and the fan. There is a diffuser behind the fan serving as controller, in order to prevent highpressure deviation from the test section part as wind turbine trial [20].

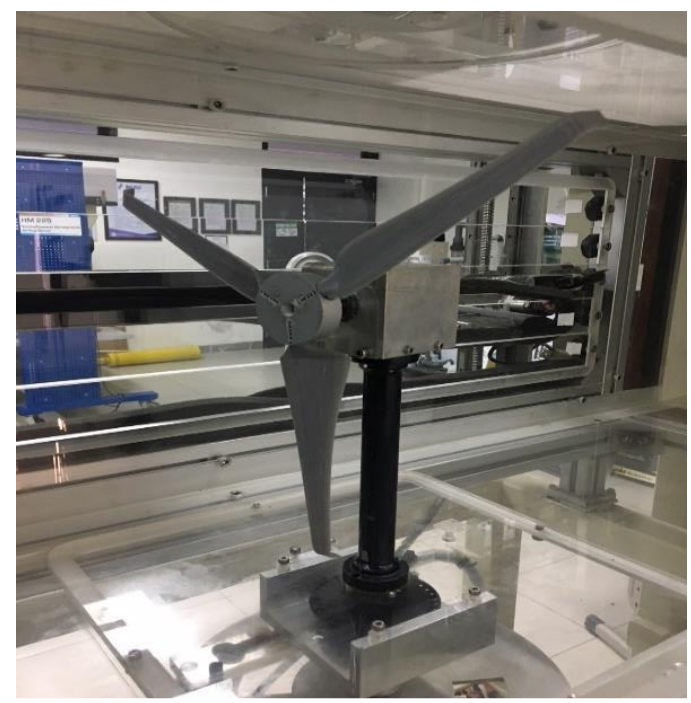

Fig. 4. The experimental process on wind tunnel.

The wind speed adjusted based on the test done using the inverter. The wind tunnel is equipped with tachometer and torquemeter that connected directly between the turbines below the rotor. It tested in computer and the data can be directly displayed and recorded on the wind turbine test stand (WTTS). The turbine tower is merge with the gearbox ratio 1:3.5. It also connected with the RPM sensor and torque semsor.

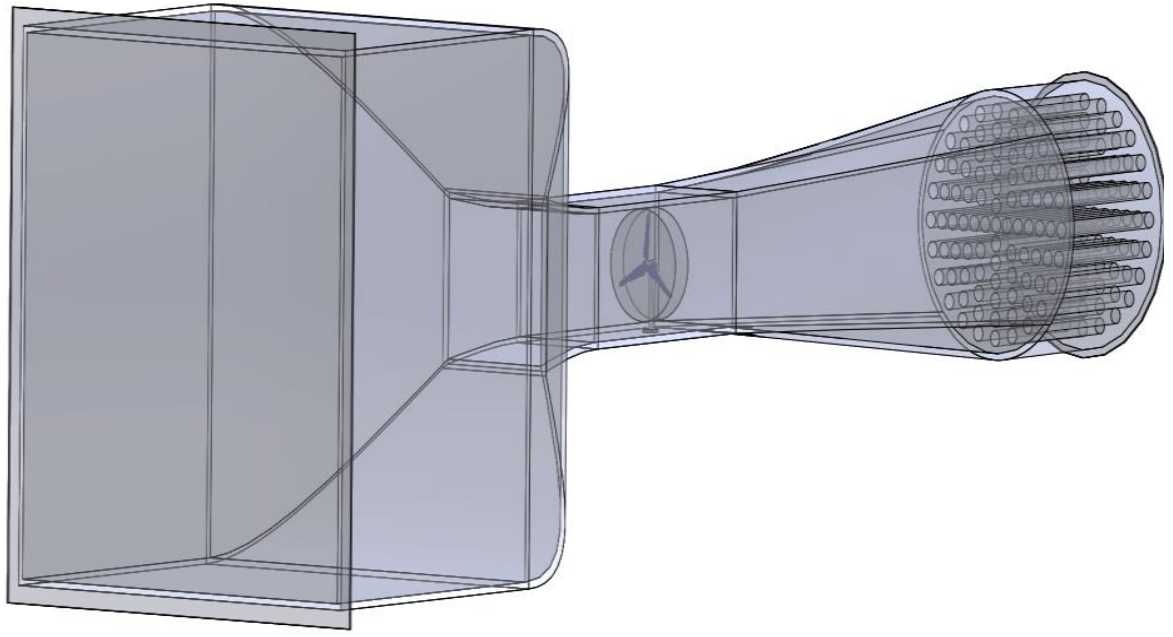

Fig. 5. The modelling of experimental setup. 
Table 1. The Specifications and configutarion of HAWT.

\begin{tabular}{lll}
\hline No. & Spesification & Description \\
\hline 1 & Rotor diameter & $31.9 \mathrm{~cm}$ \\
2 & Variation of velocity & $4 \mathrm{~m} / \mathrm{s}$ up to $7.5 \mathrm{~m} / \mathrm{s}$ \\
3 & Type of wind turbine & $\begin{array}{l}\text { Horizontal axis wind turbine } \\
\text { Clark-y, NACA 0012 with twist } \\
\text { dan tapper }\end{array}$ \\
4 & Airfoil & 3 blades \\
5 & Number of blades & Filamen hips \\
6 & Materials of blades & Blended winglets \\
7 & Type of winglet & $0^{0}$ \\
8 & Initial arrangement from & \\
& the angle of attack & $0^{0}, 2^{0}, 4^{0}, 6^{0}, 8^{0}, 0^{0}$ \\
\hline
\end{tabular}

The experiment process that conducted in wind tunnel may appear error factor caused by the area of small wind tunnel testing, hence the gap or the distance between the test object and the wall is quite close. It causes the boundary layer from the test object distracted thus the measurable result is not in accordance with the external flow assumption.

The measurable result needs to be reanalyzed again by adding correction factors of the obstacle, which is known as blockage correction factor $(\Psi)[15],[21]-[26]$, therefore, in this study the calculation of blockage factor on wind tunnel which is used to calculate the value of $l / h=10 / 30=0.3$ because $l / h, 0.5$ [26]. The equation can be written as below

$$
\Psi=1+\varnothing\left(1.11+0.94\left(\frac{-}{b}\right) C d \frac{A m}{A w}\right)
$$

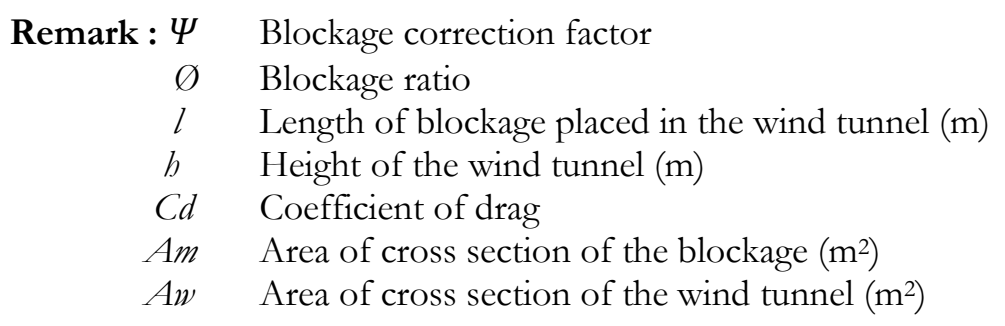

\subsection{Three Dimensional Simulation and Numerical Analysis}

CFD simulation carried out through three main steps, namely: pre-processing, processing, and solver [21]. The geometry simulation on CFD uses wind turbine rotor, which put in a block. The block defined as the wind tunnel test with the environment where the air flows. The air stream flows through an inlet, which defined on the wind tunnel input test section, while the circle volume defined as the rotation domain on the wind turbine, and the turbine rotor defined as the object pounded by the air stream. Before moving into the meshing process, each section given a name, which shows the identity on setup process. Five subsections in this study encompass input, outlet, surface section, rotational section, and HAWT [14]. 


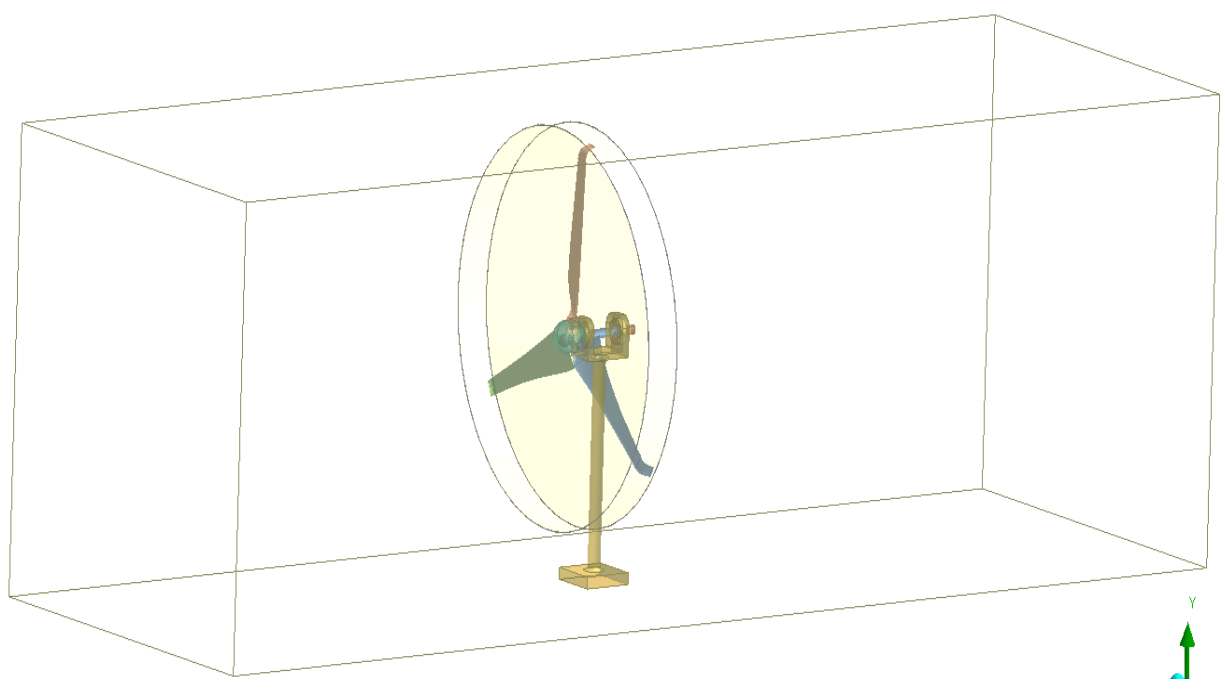

Fig. 6. The modelling of horizontal axis wind turbine with winglet.

After the modeling process, the next step is meshing. Mesh functions as the point maker for the calculation process. The calculation used to calculate element distribution on each geometry point [22]. The point distribution finished by using the tetrahedron method. The figure of mesh points or cell will be tight when they are tighter on the rotor. Inflation layer added around the airfoil to analyze layer boundary on the airfoil.
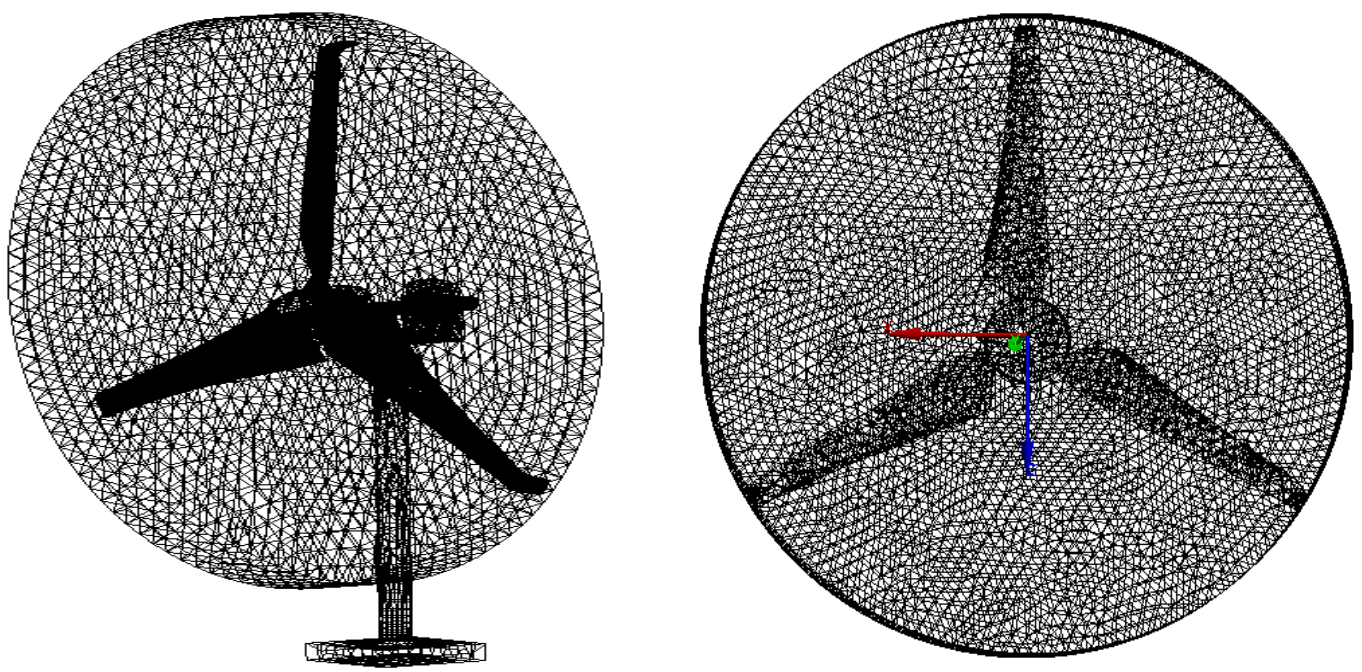

Fig. 7. Meshing process on the horizontal axis wind turbine with winglet.

The reduction of an element size is done on each domain in order to make the distribution and calculation process well processed. The mesh quality measured by using three main parameters, namely orthogonal quality, aspect ratio, and skewness [29]. A good meshing parameter does not exceed the maximum number of 0.98 based on the generating process on the meshing section.

In this research, the value of skewness obtained is 0.86 , thus the value does not exceed the value of 0.98 and can be an input for the solver process on fluent. The next step is determining the boundary condition, on the next step is regarding input boundary condition (input speed) and output boundary condition (output pressure). 
Table 2. The condition form solver on fluent.

\begin{tabular}{ll}
\hline Parameter & Description \\
\hline Turbulence model & $\begin{array}{l}\text { k- } \omega \text { shear stress transport (SST) low } \\
\text { correction Reynolds number }\end{array}$ \\
Rotating domain & Mesh motion with angular velocity \\
Boundary condition & Rotate section and test section \\
inlet & Velocity inlet normal to the boundary \\
Outlet & Pressure outlet \\
HAWT & Wall (moving rotational) \\
Symmetry & Symmetry \\
Initialization method & Hybrid Initialization \\
\hline
\end{tabular}

The calculation process utilizes fluent, where the simulation process uses the Moving Reference Frame (MRF) method. The fluid is in the form of flowing air through Wind tunnel test section inlet with standard air density. On the initial step of the solver, the modeling process on the flow characteristics was done, encompassing model turbulence using type $k-\omega S S T$.

It formulates the standard model of $k-\omega$ which is balanced and accurate on the free stream flow. $\mathrm{k}-\omega$ $S T T$ is excellent in solving the case of flow with adverse pressure gradient and high flow separation.

\section{Result and Discussion}

This section focuses on the result obtained through the process of data collection and calculation from the method result used on the horizontal axis wind turbine with winglet. The two dimensional simulation has been represented by using blade element momentum (BEM) based on the design, which was done with the total segment of 16, calculated by using the aerodynamic effect towards produced torque on each segment along the blade.

The second method is the experimental testing. The experimental setup was carried out in the wind tunnel with pitch angle variation and velocity, which was given manually from the change of pitch angle and speed. The third method is a numerical analysis, which is done by using Computational Fluid Dynamics (CFD) in order to find out the characteristics and phenomenon occurred during the turbine operation. The third method elaborates the characteristics and performance produced from the used method difference, with pitch angle variations and wind speed.

\subsection{The Experimental Result on Wind Tunnel.}

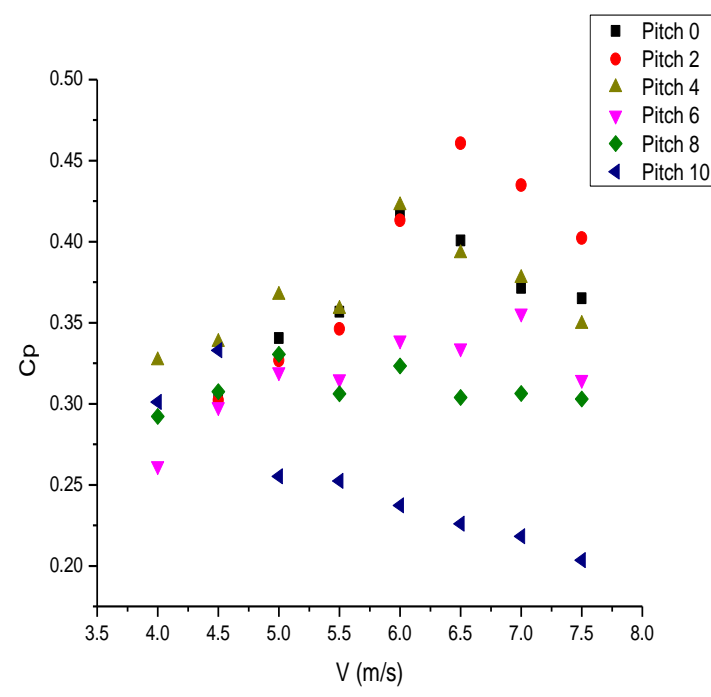

(a)

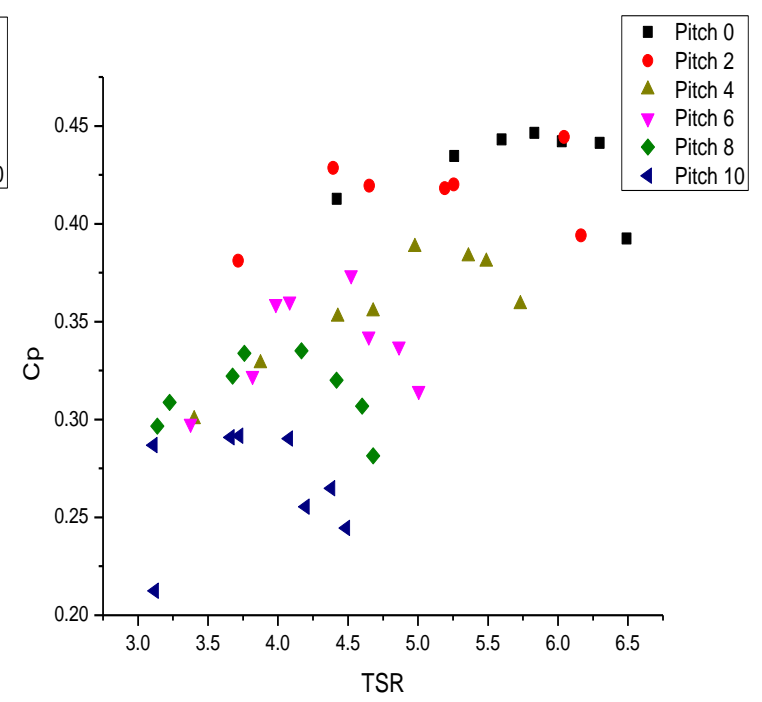

(b)

Fig. 8. The Variation of pitch angle, velocity toward coeffcient of performance on experimental. 
The addition of pitch angle variation on the test effects on different performance on a certain speed. The highest coefficient of performance was produced on the speed variation of $6.5 \mathrm{~m} / \mathrm{s}$ on pitch angle $2^{0}$. It indicates that the angle of $2^{0}$ is an effective angle in order to be varied on angle inflow projected when the speed variation is given of $6-6.5 \mathrm{~m} / \mathrm{s}$. If it is seen from the used airfoil characteristics, that is Clark- $\mathrm{Y}$, and using the utilization of maximum angle of attack of $5^{0}$. The twist design on the blade used with maximum effective Reynold number on airfoil with maximum value 140,000, allowing the $C p$ value obtained will be high, as the result from pitch effectiveness given to angle of attack adaptation which is received on the speed given.

Compared to when the wind turbine is working on the pitch variation $10^{\circ}$, the turbine can produce the highest $C P$ value than the other pitch variations on the lowest wind speed. It is caused by the speed of $4 \mathrm{~m} / \mathrm{s}$, which is the lowest speed. The test needs high starting torque to rotate the turbine, thus the pitch variation $10^{\circ}$ gives an impact that the magnification of pitch angle given gives simplicity in starting torque on low speed. The impact of winglet additions increases the starting torque as well, as the result of flow on the tip of the blade changing its direction with low-speed distribution to give the increase of lift force on each blade segment, especially the segment on the tip of the blade. The pitch angle variation is given on the wind turbine has different performance on certain TSR. The biggest impacts of pitch angle are namely the $2^{0}$ pitch variation on high TSR, $4^{0}$ pitch variation on average TSR, and $10^{\circ}$ pitch variation on low TSR. A selection criterion is because at this angle it produces the greatest $C p$ value in different TSR conditions. The highest $C p$ occurs on TSR 5.5 on pitch 20 with the value of 0.46 . TSR 5.5 explains the comparison of the amount of speed on the tip of the blade is 5.5 times of the wind speed. It means that when in the speed triangle a large inflow angle will be formed so that a pitch angle is needed to adjust the incoming angle of synchronization at the Clark-Y airfoil performance of $5^{0}$.

Based on $4^{0}$ pitch angle variation, the highest $C p$ occurs on TSR 5.41 with the value of 0.4223 . Based on maximum TSR value on low speed is obtained in $10^{\circ}$, with the highest Cp of 0.333 on TSR 3.2. It is seen that the pitch angle $2^{0}$ is better when working on high TSR but on low TSR, the wind turbine performance decreased because it does not have strong torque to rotate the turbine, on the contrary, if it is working on pitch $10^{\circ}$. It is reliable when working on low TSR because the torque produced is high along with the pitch variation, but along with the increase of TSR make the turbine performance decreased.

\subsection{Blade Element Momentum (BEM) Result}

Figure 9 shows the relationship between the coefficient of performance $(C p)$ value which is produced based on the given speed variations. It can be seen that the value consistency of $C p$ produced stands at the high value which occurs on $2^{0}$ and $4^{0}$ pitch angle variation. It indicates that $2^{0}$ and $4^{0}$ variations are the most effective variations on the change magnification of the angle of attack which is given to each wind turbine blade with given speed differences. Based on the aerodynamic aspect, the angle adapts the incoming angle of attack with a different value on the speed triangle projection produced.

However, with airfoil designation based on the Reynold numbers of 140,000, if it does not exceed that value, the consistency of the blade produces high torque is not affected at varying speeds with the provision of the given pitch angle according to the design of each wind turbine blade. The difference in values between the calculation of BEM and experimental was not far adrift, differences occur due to the experimental process carried out in the wind tunnel which does not rule out the possibility of technical and non-technical errors during the data retrieval process. 


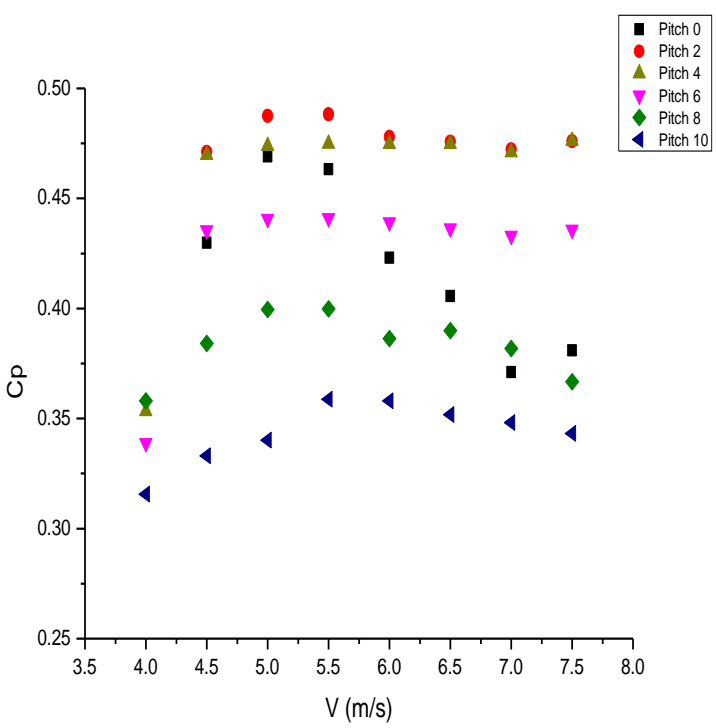

(a)

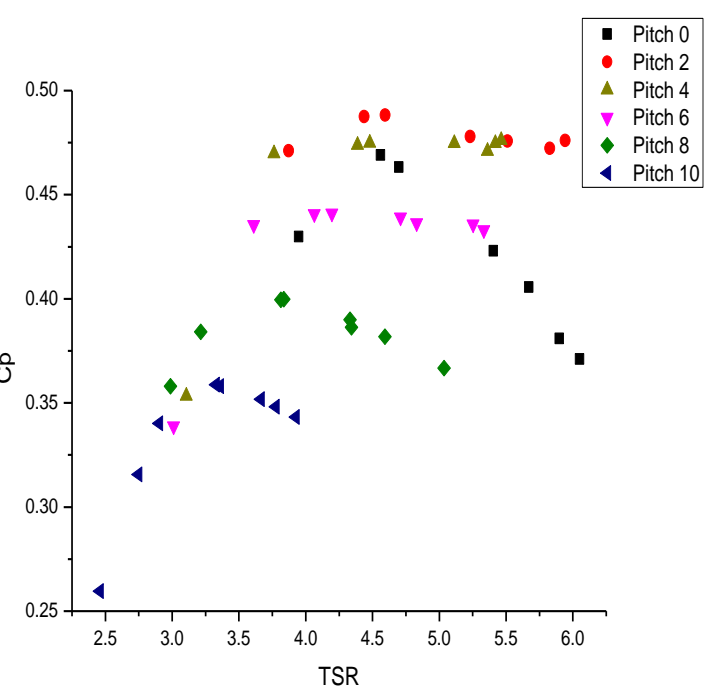

(b)

Fig. 9. The variation of pitch angle, velocity toward coeffcient of performance on blade element momentum (BEM).

Graph trends generated on pitch $0^{0}$ between experimental and BEM simulations have a BEM simulation, when exceeding TSR 4.5, the raised Cp value will go down, compare it with experimental data that shows an increasing trend to work on TSR 5.5.

However, for $C p$ values obtained the difference is not too far between experimental and BEM simulation as seen from these values, proving that load, fabrication and the type of material used at the time of testing affects the TSR obtained.

\subsection{Numerical Analysis Using Computational Fluid Dynamics}

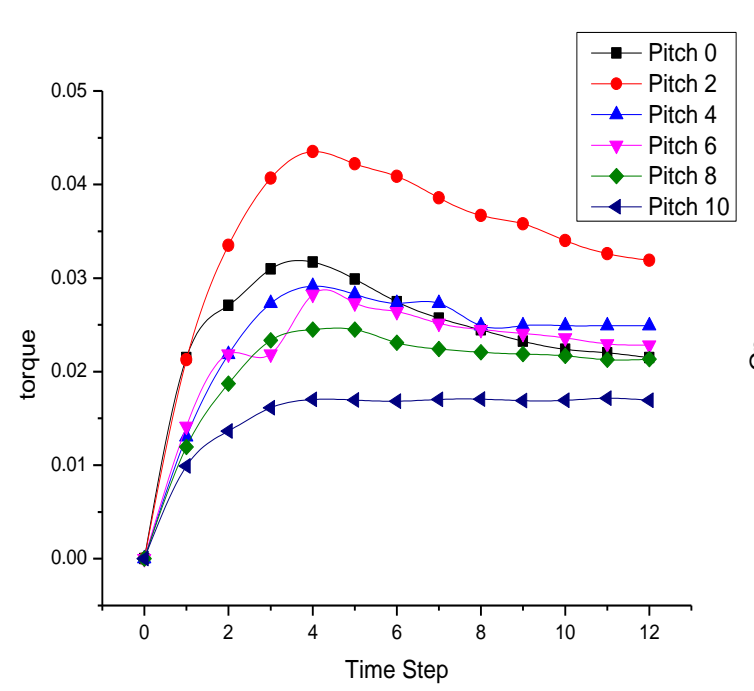

(a)

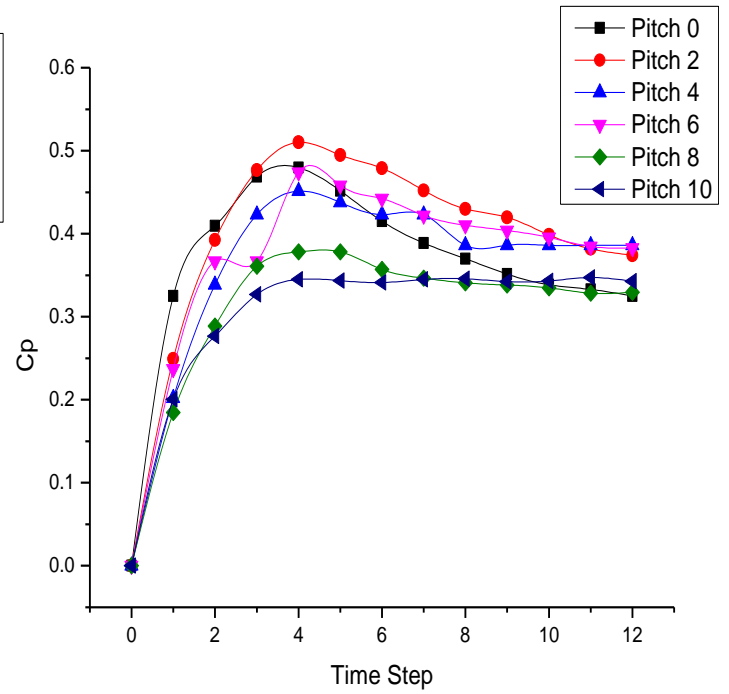

(b)

Fig. 10. The variation of pitch angle, velocity, toward coeffcient of performance on numercal analysis (CFD).

Figure 10 shows the performance of HAW'T with winglets based on the torque and coefficient of performance $(C p)$ on the rotation of the rotational rotor. It can be seen that the consistency of torque increase every time step on HAWT with winglet is longer than the time step given, especially on the lowest pitch angle, 
that is $10^{\circ}$. Lower pitch angle variations given causes the reduction of torque based on bigger time step, hence on the case of pitch variation, it proves that the magnification of pitch on each blade of HAWT gives consistency in the level of produced torque.

The variation of angle $2^{0}$ gives the highest torque performance on the use of winglet, which proves that on the use of winglets with the characteristics of airfoil Clark- $Y$ is in accordance with maximum glide ratio $(\mathrm{Cl} / \mathrm{Cd})$ on the airfoil, that is $5^{0}$ to extract the turbine rotation by producing high torque. However, on the angle $2^{0}$ shows a low probability of other pitch angle variations in terms of maintaining the increase of torque.

Figure 10 (b) shows the torque produced in optimizing the wind power which is extracted into turbine power in $\mathrm{Cp}$, in which the highest pitch angle encompasses $10^{\circ}, 8^{0}$, dan $6^{0}$, giving good consistency in the increase of $\mathrm{Cp}$ to the condition of the final azimuth angle of 12 . Those three variations are able to give an equal performance with three other angle variations $\left(0^{0}, 2^{0}\right.$, dan $\left.4^{\circ}\right)$, hence the winglet additions on the tip of the blade give good consistency towards big pitch angle variations.

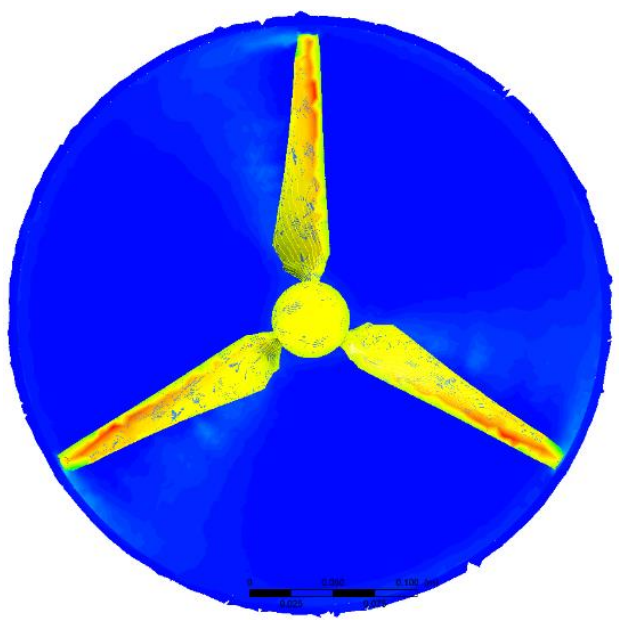

(a)

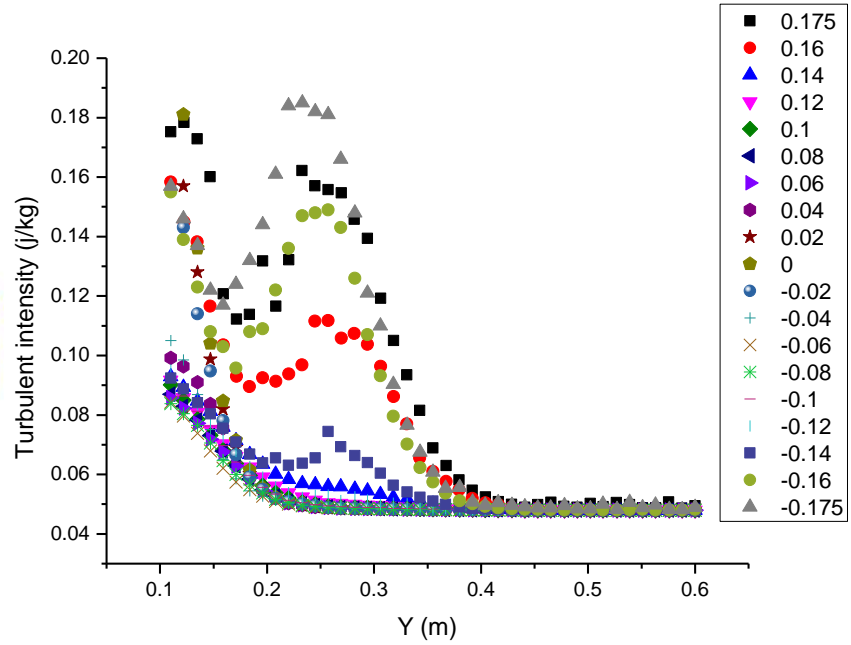

(b)

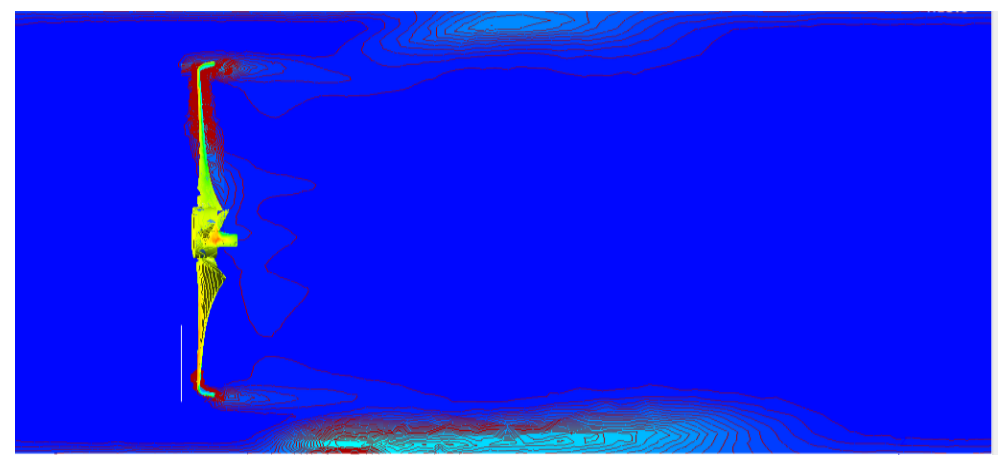

(c)

Fig. 11. Turbulent intensity contour on horizontal axis wind turbine with winglet.

Figure 11 shows the tip of the blade with winglets gives high turbulence intensity on the on the contour, but it needs to take into account that the contour is produced in high speed $(6 \mathrm{~m} / \mathrm{s})$. As the result, the winglet part gives big turbulence intensity production. It is caused by the interaction of speed distribution given to the boundary layer between wind tunnel test section and the rotor rotation, therefore the tendency on the winglet part will produce bigger turbulence intensity compared to the wind turbine without winglets because it encounters the acceleration between the wall and the turbine.

However, the addition of winglets reduces the turbulence intensity produced on other blade segments. The addition of winglet reduces the velocity on the tip of the blade caused by the interaction between winglets and the free stream velocity that results from the energy transfer process and conserved wake on a certain 
distance. The wake characteristics which have been conserved are the value of wake speed back in the input speed on the experimental process which is closing the free stream.

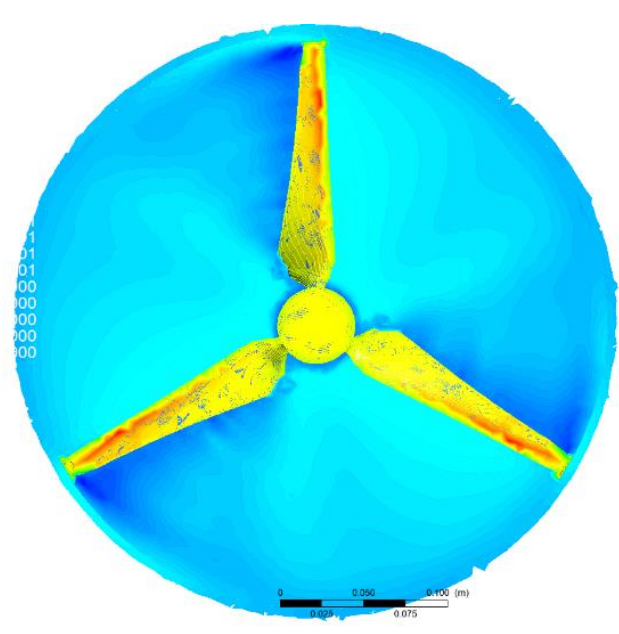

(a)

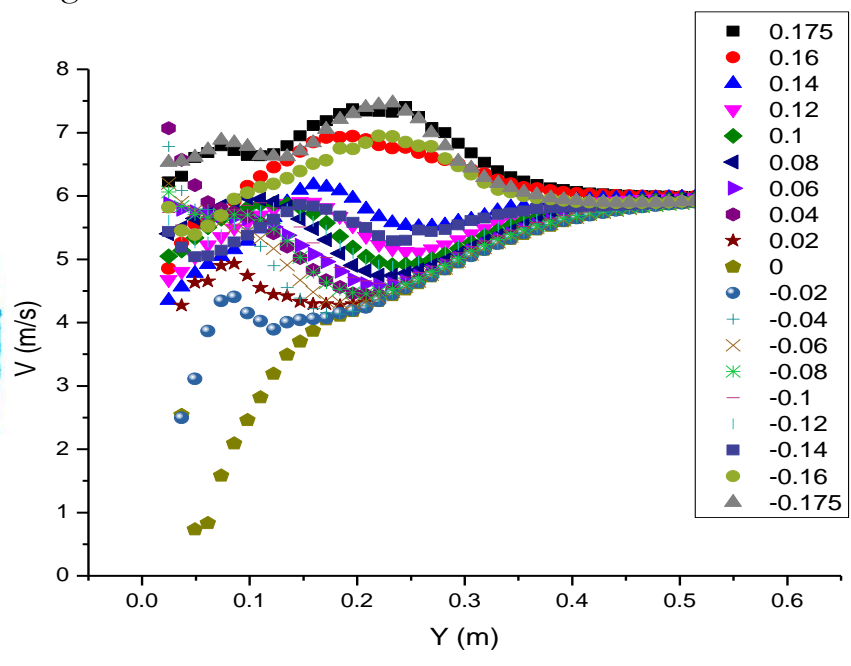

(b)

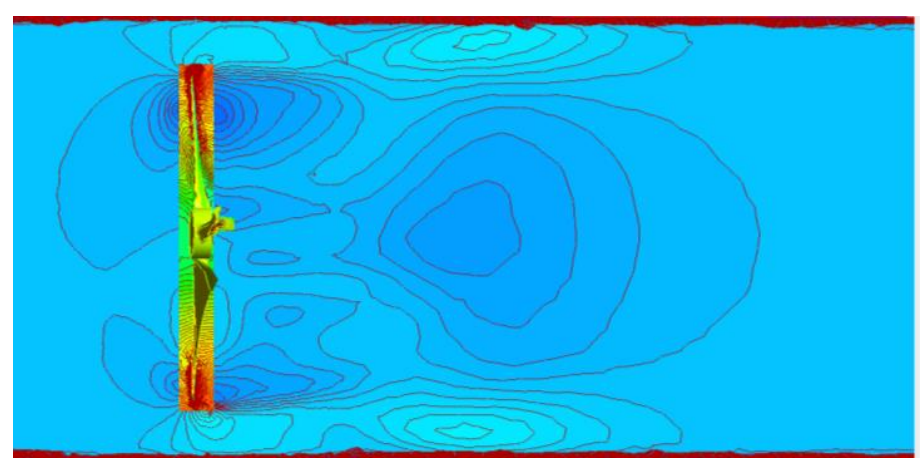

(c)

Fig. 12. Velocity contour on horizontal axis wind turbine with winglet.

The use of winglets on HAWT results a huge speed reduction based on speed flow contour. The pattern resulted on the rotation adapts the rotor rotation, therefore the winglet additions on the tip of the blade give breaking system effect if they are performing with high velocity. Nevertheless, it is necessary to consider that if the charged flow is higher, it will damage the tip of the winglet blade caused by the inability of the winglets in adapting the high-velocity pattern on the tip of the blade with the aspect ratio of winglets, which have the smallest segment length

The use of winglet on the area around the winglets resulted the velocity of $6.2 \mathrm{~m} / \mathrm{s}$ and $7.2 \mathrm{~m} / \mathrm{s}$, the velocity increase gained is caused by the interaction of rotor rotation towards test section wall and the speed on the side of inlet rotor, therefore the resulted rotation makes the freestream speed on the surface improved, as the result of the winglets as the flow splitter which cause low the velocity probability on that spot. It can be seen from the distribution chart in Fig. 12. It shows that distance $0.4 \mathrm{~m}$ on the outlet section of the wind tunnel, the current has become a fully developed. 


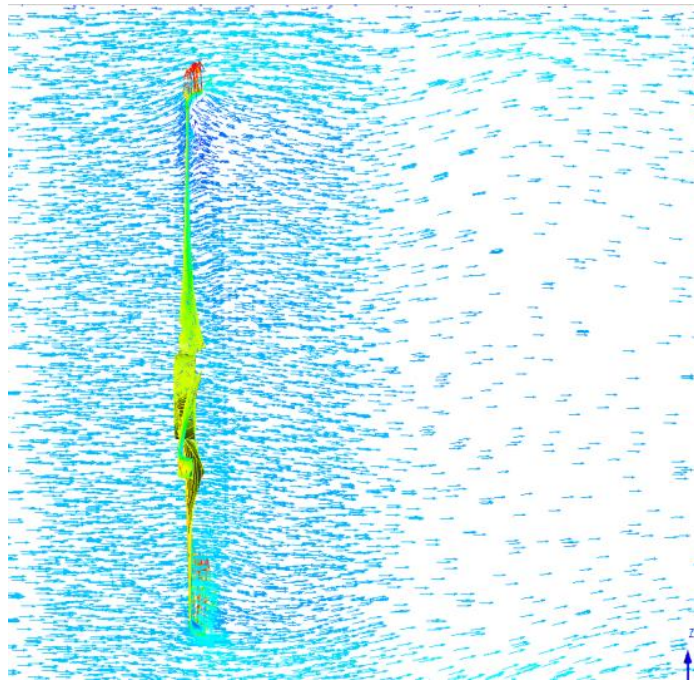

(a)

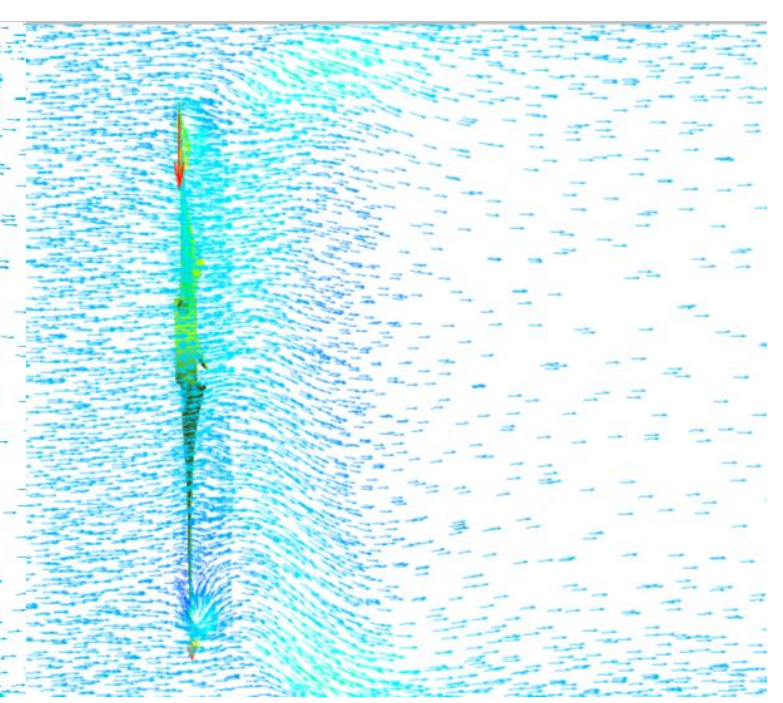

(b)

Fig. 14. Vector line on the horizontal axis wind turbine with winglet (a) and without winglet (b).

In Fig. 14 shows the velocity vector through the winglet horizontal axis wind turbine, it can be seen that the velocity vector turns direction towards the top due to the interaction of flow with the geometry of the winglet so that at the maximum speed on the airfoil in increasing the lift coefficient causes the extracted power at the end blade will increase, on the other hand with the winglet making the aspect ratio enlargement at the blade end segment allow the blade edge to be prone to deformation, this causes wind turbine testing with winglets, when the turbine works at speeds exceeding the TSR limit produced by turbine turbines causing the system to faster in receiving the stall effect due to the magnitude of the lift force obtained exceeds the glide ratio of the Clark-Y airfoil in producing lift

In wind turbines without winglets, visible at the end of the blade flow velocity vectors create an empty circle pattern at the end of the blade, so that the last segment of the blade will still produce induced drag based on increased speed and the result of a circle on the back end of the blade causing disability the edge of the blade breaks down the flow to reduce the vortex tip produced, so with the use of winglets, the resulting vortex tip can be broken which aims to reduce induced drag, but wind turbines without winglets have good reliability in terms of receiving high speeds so that at high speeds the performance provided by the two systems is more likely to wind turbines without winglets in extracting the rotor rotation into turbine power better than wind turbines with winglets.

Table 3. Comparison of the highest performance of the three methods of HAWT with winglet.

\begin{tabular}{lll}
\hline \multicolumn{3}{c}{ Horizontal Axis Wind Turbine with Winglet } \\
\hline Pitch $0^{0}$ & Torque & $\mathrm{Cp}$ \\
\cline { 2 - 3 } Experiment & 0.0222 & 0.4173 \\
BEM & 0.0225 & 0.4230 \\
CFD & 0.02201 & 0.4152 \\
\cline { 2 - 3 } Pitch $2^{0}$ & Torque & $\mathrm{Cp}$ \\
\hline Experiment & 0.0282 & 0.4710 \\
BEM & 0.0291 & 0.4756 \\
CFD & 0.0319 & 0.4789 \\
\hline Pitch 40 & Torque & $\mathrm{Cp}$ \\
Experiment & 0.0224 & 0.4319 \\
BEM & 0.0252 & 0.4747 \\
CFD & 0.0249 & 0.4379 \\
\hline
\end{tabular}




\begin{tabular}{lll}
\hline \multicolumn{3}{c}{ Horizontal Axis Wind Turbine with Winglet } \\
\hline Pitch $6^{0}$ & Torque & $\mathrm{Cp}$ \\
\cline { 2 - 3 } Experiment & 0.0207 & 0.3469 \\
BEM & 0.0268 & 0.4392 \\
CFD & 0.0228 & 0.3954 \\
\hline Pitch $8^{0}$ & Torque & $\mathrm{Cp}$ \\
\cline { 2 - 3 } Experiment & 0.0214 & 0.3380 \\
BEM & 0.0256 & 0.3863 \\
CFD & 0.0213 & 0.3568 \\
\hline Pitch $10^{0}$ & Torque & $\mathrm{Cp}$ \\
Experiment & 0.0168 & 0.3405 \\
BEM & 0.0181 & 0.3590 \\
CFD & 0.0169 & 0.3429 \\
\hline
\end{tabular}

Comparison of the horizontal axis wind turbine with winglet performance against maximum performance at each pitch angle variation is present in Table 3. Comparisons given to torque and $C p$ generated in each method show the percentage difference in each method, for torque the value between $0.05 \%-1.17 \%$, while for $C p$ produced has a percentage difference of $1 \%-8 \%$.

This indicates that the calculation between the three methods has high accuracy, both in the numerical calculation process of increasing torque of each segment using BEM, experimental testing in wind tunnel, and numerical simulations on CFD.

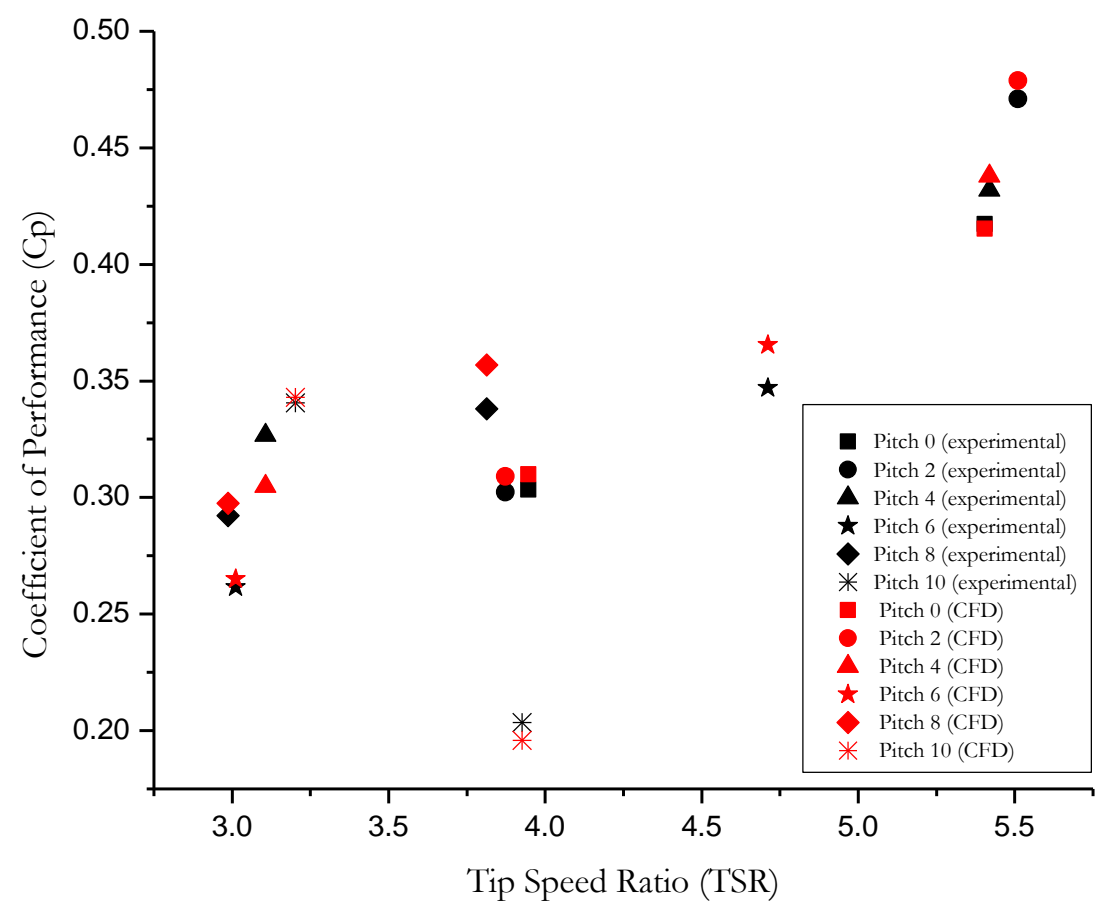

Fig. 15. Comparison between experimental result and CFD result on the horizontal axis wind turbine with winglets.

The Validation process carried out on experimental results of the horizontal axis wind turbines with winglets and it was modeled on CFD (computational fluid dynamics) with the similarity testing and conditioning metrics, wherein Fig. 15 describes the maximum and the minimum values of the tests based on pitch angle variations with metrics testing that gives maximum and minimum performance and results to the generated $C p$ value. 
The errors generated in this validation ranged from $0.67 \%-5.28 \%$ for maximum performance and $0.51 \%$ - $5.58 \%$ for minimum performance in winglet horizontal axis wind turbines, this indicates that the modeling performed on the same metrics and conditioning approached the results of the direct implementation with an error factor of $<10 \%$.

\section{Conclusions}

The pitch angle addition as a variation influences the performance of wind turbine with winglets based on the gained rotating speed and the produced torque. The impact of pitch angle, which given to the winglet wind turbine, gives maximum performance on each TSR, with the pitch effectiveness of $2^{0}$ on high TSR, $4^{0}$ on average TSR and $10^{\circ}$ on low TSR. The configuration of pitch angle is adjustment results from simplicity in terms of the adjustment of the effective angle of attack on the highest value of $\mathrm{Cl} / \mathrm{Cd}$, therefore the effectiveness of lift force is higher than the increase of produced drag force. It is supported by the addition of design optimization on the blades that is the use of twist, it can cause the adjustment on segments arranging the blade that composed by same airfoil types and give a simplicity in the global adjustment hence the pitch adjustment can be set to achieve maximum performance. However, the addition of winglet on the tip of the turbine blade gives good performance when it is working in low TSR. The role of the winglet on low TSR increases the starting torque during the test with low rotation. The effectiveness of winglet use is also good on pitch variation is given with the correlation of speed and TSR variations which were charged and produced.

\section{Acknowledgements}

The authors would like to gratefully thank Fluid Mechanics Laboratory and Energy Engineering and Environmental Conditioning Laboratory for their support to provides the facility and materials. Thanks also go to the LPPM ITS for funding this research.

\section{References}

[1] U. Chaudhary and S. K. Nayak, "Micro and small-scale HAWT blades airfoils study through CFD for low wind applications," in 12th International Conference Electronic Energy Environmental Communication Computer. and Control, 2016, vol. 1, pp. 1-6.

[2] J. E. Guerrero, D. Maestro, and A. Bottaro, "Biomimetic spiroid winglets for lift and drag control," Journal of Comptes Rendus - Mecanique, vol. 340, pp. 67-80, 2012.

[3] B. Zhu, X. Sun, Y. Wang, and D. Huang, "Performance characteristics of a horizontal axis turbine with fusion winglet," Journal of Energy, vol. 120, pp. 431-440, 2017.

[4] S. Lawton and C. Crawford, "Investigation and optimization of blade tip winglets using an implicit free wake vortex method," Journal of Physics, Vol. 524, pp. 1-13, 2014.

[5] G. Narayan and B. John, "Effect of winglets induced tip vortex structure on the performance of subsonic wings," Journal of Aerospace Science and Technology, vol. 58, pp. 328-340, 2016.

[6] J. M. O’Brien, T. M. Young, J. M. Early, and P. C. Griffin, "An assessment of commercial CFD turbulence models for near wake HAWT modelling," Journal of Wind Engineering and Industrial Aerodynamic, vol. 176, no. February, pp. 32-53, 2018.

[7] M. N. Kaya, F. Kose, D. Ingham, L. Ma, and M. Pourkashanian, "Aerodynamic performance of a horizontal axis wind turbine with forward and backward swept blades," Journal of Wind Engineering and Industrial Aerodynamic, vol. 176, no. December 2017, pp. 166-173, 2018.

[8] S. A. Abdulqadir, H. Iacovides, and A. Nasser, "The physical modelling and aerodynamics of turbulent flows around horizontal axis wind turbines," Journal of Energy, vol. 119, pp. 767-799, 2017.

[9] Y. El khchine and M. Sriti, "Tip loss factor effects on aerodynamic performances of horizontal axis wind turbine," Journal of Energy, vol. 118, pp. 136-140, 2017.

[10] W. D. Omoware, A. Maheri, and U. Azimov, "Aerodynamic analysis of flapping-pitching flat plates," presented at 3rd International. Symposium. Environmental Friendly Energies Applied, 2014.

[11] M. M. Oueslati, A. W. Dahmouni, and S. Ben Nasrallah, "Numerical study of pitching wind turbine airfoil," presented International Conference of Composite Material and Renewable Energy Applied, 2014.

[12] D. C. Vega, J. A. Marin, and R. T. Sanchez, "Pitch angle controllers design for a horizontal axis wind turbine," presented at International Autumn Meeting on Power Electronics and Computing, 2015.

[13] E Koc, O. Gunel, and T. Yavuz, "Scaled horizontal axis wind turbine analysis," in 5th International 
Conference on Renewable Energy Research and Applications, 2016, vol. 5.

[14] J. Naima, R. Mohammed, and E. L. F. Benaissa, "Three-dimensional modeling of a horizontal axis wind turbine blade and profile effect analysis," in International Renewable and Sustainable Energy Conference, 2016, vol. 1, pp. 1-5.

[15] I. Bayati, M. Belloli, L. Bernini, and A. Zasso, "Aerodynamic design methodology for wind tunnel tests of wind turbine rotors," Journal of Wind Engineering and Industrial Aerodynamic, vol. 167, pp. 217 227, 2017.

[16] C. Yİ̆̈It and U. Durmaz, "Wind turbine blade design with computational fluid dynamics analysis," International Journal of Computational and Experimental Science and Engineering, vol. 3, no. 2, pp. 44-49, 2017.

[17] M. Tahani, G. Kavari, M. Masdari, and M. Mirhosseini, "Aerodynamic design of horizontal axis wind turbine with innovative local linearization of chord and twist distributions," Journal of Energy, vol. 131, pp. 78-91, 2017.

[18] C. Suvanjumrat, "Comparison of Turbulence models for flow past NACA 0015 using open foam," Engineering Journal, vol. 21, no. 6, pp. 208-221, 2017.

[19] E. L. C. Arrieta, C. C. Mancilla, J. Slayton, F. Romero, E. Torres, S. Agudelo, J. Arbelaez, and D. Hincapie, "Experimental investigations and CFD simulations of the blade section pitch angle effect on the performance of a horizontal-axis hydrokinetic turbine," Engineering Journal, vol. 22, no. 5, pp. 142-154, 2018.

[20] D. Damljanovic, J. Isakovic, and M. Milos, "Wind tunnel measurement quality in testing of a standard model," Materials Today Proceedings, vol. 4, no. 5, pp. 5791-5796, 2017.

[21] C. J. Bai and W. C. Wang, "Review of computational and experimental approaches to analysis of aerodynamic performance in horizontal-axis wind turbines (HAWTs)," Joumal of Renewable and Sustainable Energy Review., vol. 63, pp. 506-519, 2016.

[22] J. M. O’Brien, T. M. Young, D. C. O’Mahoney, and P. C. Griffin, "Horizontal axis wind turbine research: A review of commercial CFD, FE codes and experimental practices," Journal of Progress in Aerospace Sciences, vol. 92, no. 5, pp. 1-24, 2017.

[23] C. Hcini, E. Abidi, B. Kamoun, and D. Afungchui, "Numerical prediction for the aerodynamic performance of turbosail type wind turbine using a vortex model," Journal of Energy, vol. 109, pp. 287 293, 2016.

[24] Q. Li, J. Murata, M. Endo, T. Maeda, and Y. Kamada, "Experimental and numerical investigation of the effect of turbulent inflow on a horizontal axis wind turbine (part II: wake characteristics)," Journal of Energy, vol. 113, pp. 1304-1315, 2016.

[25] G. Bangga, G. Hutomo, R. Wiranegara, and H. Sasongko, "Numerical study on a single bladed vertical axis wind turbine under dynamic stall," Journal of Mechanical Sciences and Technology, vol. 31, no. 1, pp. 261-267, 2017.

[26] T. Y. Chen and L. R. Liou, "Blockage corrections in wind tunnel tests of small horizontal-axis wind turbines," Journal of Experimental Thermal and Fluid Sciences, vol. 35, no. 3, pp. 565-569, 2011.

[27] M. A. Abdelrahman, O. E. Abdellatif, M. Moawed, A. Eliwa, and S. Misak, "The CFD performance analysis for horizontal axis wind turbine with different blade shapes and tower effect," in 16th International Sciences Conference Electrical Power Engineering, 2015, vol. 16, pp. 754-759.

[28] F. Hsiao, C. Bai, and W. Chong, "The performance test of three different horizontal axis wind turbine (HAWT) blade shapes using experimental and numerical methods," Energies, vol. 6, pp. 2784-2803, 2013.

[29] H. Wang, B. Ma, J. Ding, and S. Li, "The modeling and stress analysis of wind turbine blade," Telkomnika Indonesia Journal of Electrical Engineering, vol. 12, no. 6, pp. 4178-4183, 2014. 